\title{
Role of integrin-linked kinase in drug resistance of lung cancer
}

This article was published in the following Dove Press journal:

OncoTargets and Therapy

23 June 2015

Number of times this article has been viewed

Zhiyang Jia

Department of Imaging and Nuclear Medicine, The First Affiliated Hospital of Zhengzhou University, Zhengzhou City, People's Republic of China
Correspondence: Zhiyang Jia

Department of Imaging and Nuclear Medicine, The First Affiliated Hospital of Zhengzhou University, Zhengzhou City 420052, People's Republic of China

Tel +86 I36 33832898

Email zhiyangjiababy@163.com
Objective: The objective of the present investigation was to investigate the role of integrin-linked kinase (ILK) in the gemcitabine-resistant lung cancer cell line A549 and explore the underlying mechanism.

Materials and methods: Gemcitabine-resistant A549 (A549/GemR) cell line was established by pulse-exposed to moderate concentration of gemcitabine (Gem), and the drug resistant index was measured by MTT assay. Expression of ILK in A549/GemR cell line was detected by Western blot and real-time PCR. An ILK gene-silencing cell line was constructed using lentivirus-coated ILK shRNA. MTT assay was used to detect the drug sensitivity of the A549/ GemR cell line to Gem after the ILK gene silencing. Western blot was used to measure the expression of E-cadherin, fibronectin, and MRP1 (multidrug resistance-associated protein 1) after silencing the $I L K$ gene.

Result: The drug resistance index of A549/GemR was 13.5, and the messenger RNA and protein level of ILK was increased in $\mathrm{A} 549 / \mathrm{GemR}$. $\mathrm{IC}_{50}$ (half maximal inhibitory concentration) decreased from 14.69 to $4.13 \mathrm{mg} / \mathrm{L}$ when ILK was knocked down in A549/GemR. The expression of fibronectin and MRP1 was upregulated and E-cadherin expression was downregulated in A549/GemR, and these changes were reversed after ILK was knocked down.

Conclusion: ILK was involved in drug resistance to Gem in lung cancer, and this function may be mediated by epithelial-mesenchymal transition and the MRP1 pathway.

Keywords: lung cancer, drug resistance, gemcitabine, ILK, EMT

\section{Introduction}

Lung cancer is a malignant tumor that causes high mortality and seriously threatens people's health. ${ }^{1}$ With the increasing number of antitumor drugs, the drug resistance of the tumor gradually becomes the main issue, which has attracted people's attention. Drug resistance is related with many factors such as impairment of drug delivery, increased drug efflux, drug inactivation by detoxifying factors, increased damage repair, tolerance of damage, gene mutation, epithelial-mesenchymal transition (EMT), and cancer stem cells (CSCs). ${ }^{2-5}$ In recent years, scholars have found that the remodeling of the tumor extracellular matrix (ECM) was also the main reason leading to survival of tumor cells from ionizing radiation and chemotherapy. ${ }^{6}$ This study tentatively discusses the role of integrin-linked kinase (ILK) in drug-resistant lung cancer and preliminarily explores the mechanism. The findings of this study might help to provide a new insight on the treatment of drug-resistant lung cancer.

\section{Materials and methods Materials}

Human lung cancer cell line A549 was purchased from the Cell Bank of Shanghai (Shanghai, People's Republic of China). Dubecco's Modified Eagle's Medium 
(DMEM) and fetal bovine serum were purchased from Hyclone (GE Healthcare Bio-Sciences Corp., Piscataway, NJ, USA). Gemcitabine (Jianze), primer synthesis (Huada Gene, Shenzhen, People's Republic of China), Trizol reagent (Thermo Fisher Scientific, Waltham, MA, USA); real-time PCR reagent (Takara Bio Inc., Otsu, Japan), fibronectin (FN) antibody (Sigma-Aldrich Co., St Louis, MO, USA), ILK, E-cadherin, and MRP1 antibody (Abcam, Cambridge, UK), GAPDH, goat anti-mouse IgG and goat anti-rabbit IgG (Beijing ZSGB-BIO GRIGENE, Beijing, People's Republic of China). MTT (thiazole blue) was purchased from Beyotime Institute of Biotechnology (Haimen, Jiangsu, People's Republic of China); lentivirus coated ILK shRNA (Henan Biotools Biological Technology Co., LTD, Henan, People's Republic of China) was also purchased.

\section{Methods}

\section{Establishment of A549 drug resistant cell line} (A549/GemR)

A549 cells in the logarithmic phase of growth were seeded on six-well plates, and $2 \mathrm{~mL}$ DMEM was added with $1.5 \mathrm{mg} / \mathrm{L}$ gemcitabine hydrochloride. After 48 hours (h), the DMEM was replaced by the fresh medium without gemcitabine hydrochloride. Fresh medium was added once every 2 days, and the above steps were repeated until the cells recovered. The above steps were repeated, with the concentration of the drug gradually increasing between the generations, and this treatment was continued for ten generations.

\section{MTT assay}

Logarithmically growing A549 or A549/GemR cells were seeded on a 96 -well plate $\left(5 \times 10^{3} /\right.$ well $)$ and cultured for $24 \mathrm{~h}$ at $37^{\circ} \mathrm{C}$ in a $5 \% \mathrm{CO}_{2}$ incubator. Then, proper concentration of gemcitabine hydrochloride was added and incubated for $48 \mathrm{~h}$. Cells were incubated for another $4 \mathrm{~h}$ in the presence of $10 \mu \mathrm{L}$ MTT ( $5 \mathrm{mg} / \mathrm{mL}$ ), and then the medium was discarded and $200 \mu \mathrm{L}$ DMSO was added into every well. The spectrophotometric absorbance was measured at $490 \mathrm{~nm}$ with enzyme-labeling instrument after the crystals were fully dissolved.

\section{The construction of A549/GemR ILK-silencing cell}

Logarithmically growing A549/GemR cells were seeded on 24-well plates and cultured for $24 \mathrm{~h}$ at $37^{\circ} \mathrm{C}$ in a $5 \% \mathrm{CO}_{2}$ incubator. Then, lentivirus-coated shRNA control and ILK shRNA were added. The medium was replaced with fresh medium after incubation for $12 \mathrm{~h}$, and after $72 \mathrm{~h}$, puromycin was added for selecting the positive cells.
The expression of ILK messenger ( $m$ )RNA was detected by real-time PCR

The mRNA was isolated from the cells using Trizol reagent, the reverse transcriptase reagent kit (Takara) was used to obtain the cDNA, and the expression of ILK mRNA was measured by real-time quantitative PCR. ILK-forward primer: 5'-TGGAACCCTGAACAAACACTC-3', ILK-reverse primer: 5'-AGCACCTTCACGACAATGTCA-3', and GAPDH forwarder primer: 5'-CTGGGCTACACTGAGCACC-3', GAPDH reverse primer: 5'-AAGTGGTCGTTGAGGGCAATG-3' were used.

\section{Western blot}

Cells were washed twice with precooled PBS after discarding the medium, and the total protein was extracted with RIPA lysis buffer containing protease inhibitor. The protein was separated through $8 \%$ SDS-PAGE and then transferred to PVDF membrane (EMD Millipore, Billerica, MA, USA). Membranes were blocked with 5\% skimmed milk powder for $2 \mathrm{~h}$ and incubated with proper antibodies overnight at $4{ }^{\circ} \mathrm{C}$. The membranes were washed with TBST buffer three times and then incubated with the second antibody for $2 \mathrm{~h}$ at room temperature. The membrane was washed, and the protein level was detected by electrochemiluminescence method.

\section{Statistical analysis}

Data were analyzed with SPSS 13.0 (SPSS Inc., Chicago, IL, USA) and Graphpad Prism software (La Jolla, CA 92037 USA). All data were expressed as mean \pm standard deviation (SD); Student's $t$-test was used for statistical analysis. Difference with $P<0.05$ was considered statistically significant.

\section{Results}

\section{Drug resistance index of A549/GemR was significantly higher than that of A549}

Logarithmically growing A549 and A549/GemR cells were seeded on 96-well plates and different concentrations of gemcitabine were added. MTT cell proliferation assay was used to detect the drug resistance. Figure 1 revealed that the $\mathrm{IC}_{50}$ (half maximal inhibitory concentration) of A549/GemR cells $\left(\mathrm{IC}_{50}=18.08 \mathrm{mg} / \mathrm{L}\right)$ was significantly higher than that of A549 cells $\left(\mathrm{IC}_{50}=1.34 \mathrm{mg} / \mathrm{L}\right)$. The drug resistance index was 13.5, which suggested the construct of A549/GemR was successful.

\section{Expression of ILK in A549/GemR cells was upregulated}

Total RNA and protein of A549 and A549/GemR cells were extracted, then real-time PCR (Figure 2A) and Western blot 


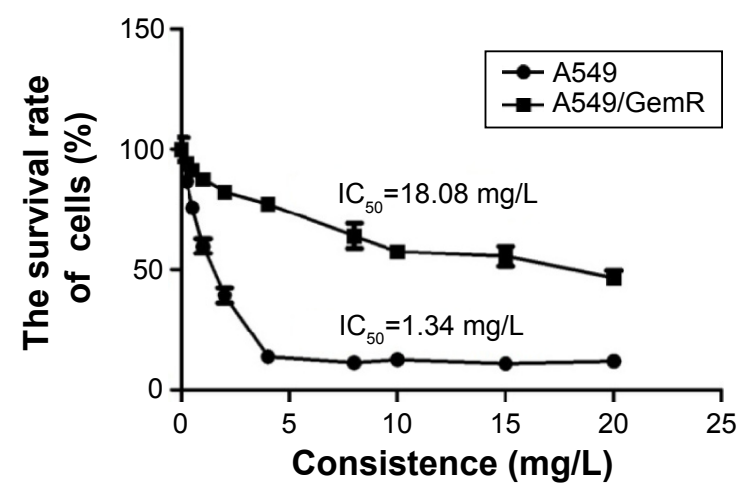

Figure I Survival rates of A549/GemR and A549 cell lines.

Abbreviations: GemR, gemcitabine resistant; $\mathrm{IC}_{50}$, half maximal inhibitory concentration.

(Figure 2B) were performed to detect the expression of ILK. From Figure 2, we can deduce that the mRNA and protein level of ILK were significantly higher in the drug resistantA549/GemR cell line.

\section{Knock down of ILK gene causes A549/ GemR cells to be sensitive to gemcitabine} In order to further confirm the function of ILK in A549 cell resistance, the lentivirus-coated ILK shRNA was used to infect A549/GemR cells, and the silencing efficiency was validated by Western blot (Figure 3A). The results of MTT cell proliferation assay revealed that drug resistance of A549/GemR cells decreased when ILK gene was silenced (Figure 3B). The $\mathrm{IC}_{50}$ of A549/GemR cells had decreased from 14.69 to $4.13 \mathrm{mg} / \mathrm{L}$.

\section{Molecular mechanism of ILK in gemcitabine resistance}

Western blot results showed that expression of the mesenchymal marker FN was obviously increased in A549/ GemR cells, while the level of epithelial marker E-cadherin decreased remarkably. However, when ILK was silenced, the expression of FN decreased, and the expression of E-cadherin increased (Figure 4A). We also found that the expression of MRP1, which mediated export of organic anions and drugs from cells, was elevated in gemcitabine-resistant cells. This increase was weakened when ILK was knocked down with RNA interference (Figure 4B). This finding suggested that ILK might be regulating drug resistance of lung cancer by promoting EMT and cellular drug efflux.

\section{Discussion}

Tumor drug resistance has received more and more attention despite the exact mechanism still being unclear. Studies have proven that the expression of drug transport-related proteins was obviously increased in drug-resistant cells, ${ }^{7}$ and the resistance of apoptosis was reinforced. ${ }^{8}$ In recent years, some studies revealed that tumor drug resistance was related to the remodeling of the ECM, EMT progress, and tumor stem cells. ${ }^{9,10}$ In this study, we found that the progress of EMT was reinforced in the gemcitabine-resistant lung cancer cell line A549/GemR cells.

ILK, an important serine/threonine protein phosphatase, plays a key role in the regulation of signal transduction and the remodeling of the tumor ECM. ${ }^{11}$ High expression of ILK was closely related to the occurrence and development of gastrointestinal, breast, and lung tumors. ${ }^{12-15}$ A study by Chen et al showed that ILK could promote the process of lung cancer EMT, and silencing of ILK gene could significantly inhibit the invasion and metastasis of lung cancer. ${ }^{10}$ This conclusion was also confirmed in melanoma and breast cancer. ${ }^{16,17}$ Tabe et al showed that the activity of ILK and AKT was enhanced in the lymphoma cells, which indicated that ILK may also play an important role in CSCs. Gemcitabine is a pyrimidine nucleoside antimetabolite agent which is active in several
A

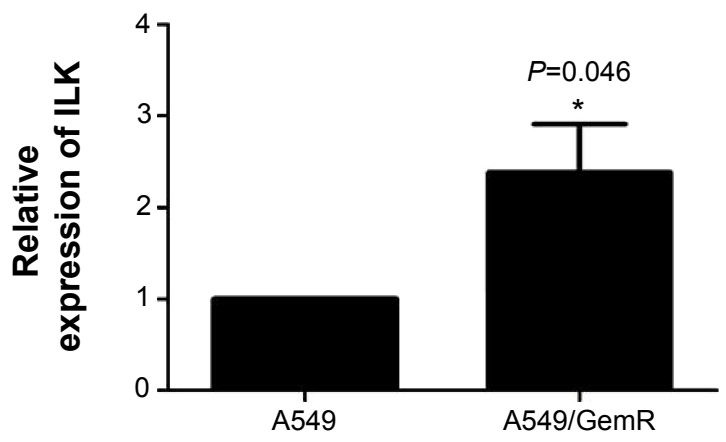

B

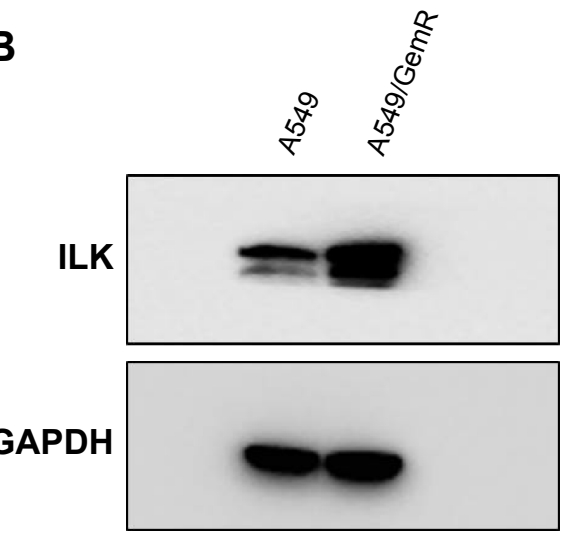

Figure 2 The relative expression of ILK in A549/GemR and A549 cells.

Notes: (A) The messenger RNA expression of ILK. (B) The protein expression of ILK.

Abbreviations: ILK, integrin-linked kinase; GemR, gemcitabine resistant. 
A

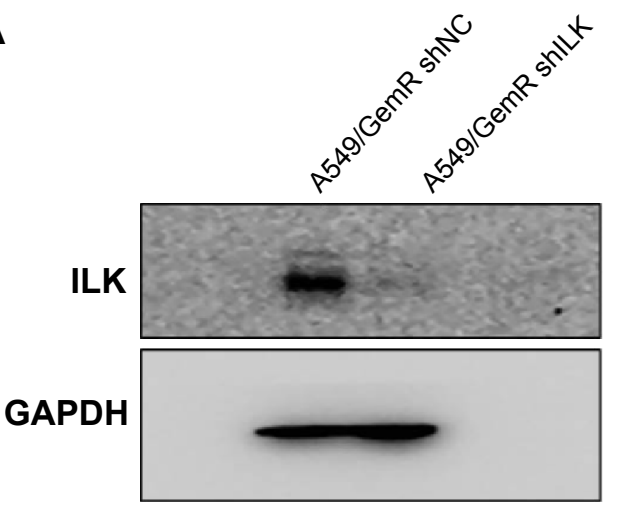

B

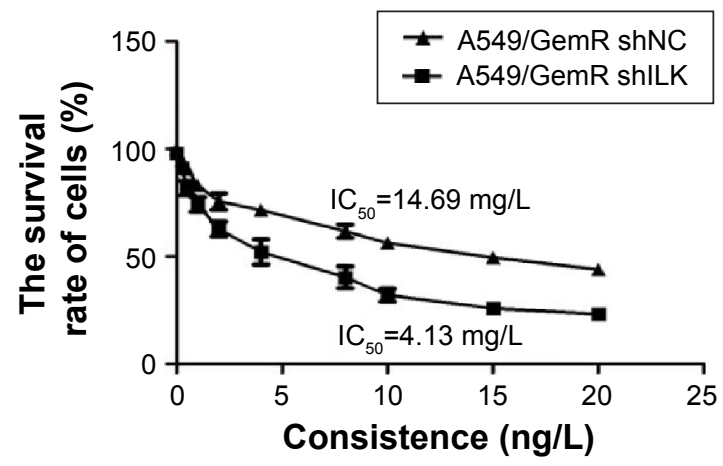

Figure 3 The function of ILK in gemcitabine resistance.

Notes: (A) The expression of ILK in A549/GemR shILK cells. (B) The survival rate of A549/GemR shlLK cells.

Abbreviations: ILK, integrin-linked kinase; GemR, gemcitabine resistant; ILK, integrin-linked kinase.

human malignancies, including non-small-cell lung cancer (NSCLC). ${ }^{18,19}$ However, gemcitabine resistance was found in the chemotherapy of cancers recently. Our research first verified that the mRNA and protein expression of ILK were increased in gemcitabine-resistant A549 cell lines. When ILK gene got silenced by RNA interference technology, the drug resistance recovered to a certain degree. These results preliminarily proved that ILK was involved in the drug resistance of lung cancer. Our findings added some novel information to the function of ILK in cancer.

Considering EMT process played an important role in tumor drug resistance, and ILK was involved in cell survival, EMT progress and maintenance of tumor stem cells. So, the related indexes of EMT were tested in this study. As expected, the results showed that FN was increased in drug-resistant cells, while the epithelial marker E-cadherin was decreased. This phenomenon got reversed when ILK gene was silenced, which suggested that the occurrence of EMT process in tumor drug resistance may be mediated by the ILK.

MRP1, a member of the ATP-binding cassette (ABC) transporter superfamily, was discovered as a cause of multidrug resistance in tumor. High level of MRP1 is related to drug resistance and metastasis of cancer. ${ }^{20} \mathrm{~A}$ recent study confirmed that MRP1 played a prominent role in the efflux of gemcitabine. ${ }^{21}$ In this paper, we revealed that ILK was a regulator of MRP1; silence of ILK in gemcitabine-resistant lung cancer cell line could significantly decrease the elevated levels of MRP1.
A

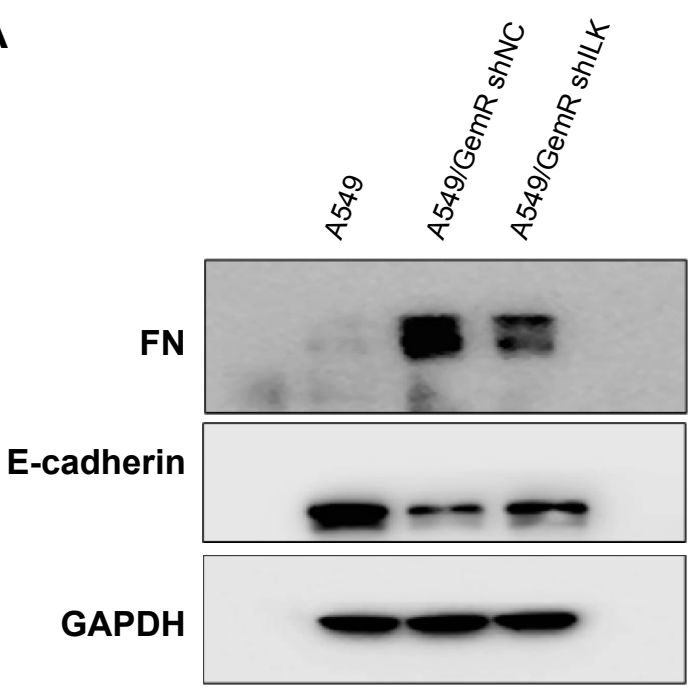

B

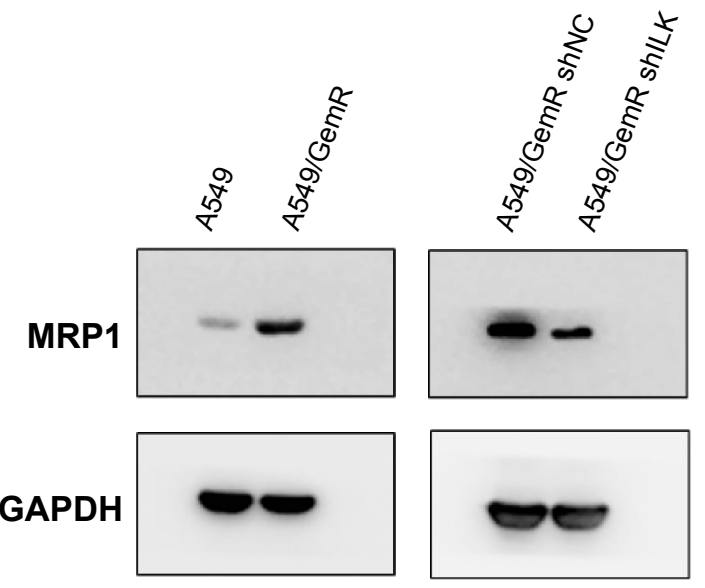

Figure 4 The molecular mechanism of ILK in gemcitabine-resistant lung cancer.

Notes: (A) The markers of EMT were detected by Western blot. (B) The expression of MRPI was detected by Western blot.

Abbreviations: ILK, integrin-linked kinase; EMT, epithelial-mesenchymal transition; MRPI, multidrug resistant protein I; FN, fibronectin; GemR, gemcitabine resistant. 
In summary, this study confirmed that ILK was involved in drug-resistant lung cancer, and this function might be mediated by EMT process and MRP1 pathway. This provided new proof and a new direction for the treatment for tumor drug resistance based on ILK.

\section{Disclosure}

The author reports no conflicts of interest in this work.

\section{References}

1. Siegel R, DeSantis C, Virgo K, et al. Cancer treatment and survivorship statistics, 2012. CA Cancer J Clin. 2012;62(4):220-241.

2. Stewart DJ. Tumor and host factors that may limit efficacy of chemotherapy in non-small cell and small cell lung cancer. Crit Rev Oncol Hematol. 2010;75(3):173-234.

3. Oxnard GR, Arcila ME, Chmielecki J, et al. New strategies in overcoming acquired resistance to epidermal growth factor receptor tyrosine kinase inhibitors in lung cancer. Clin Cancer Res. 2011;17(17):5530-5537.

4. Goffin JR, Zbuk K. Epidermal growth factor receptor: pathway, therapies, and pipeline. Clin Ther. 2013;35(9):1282-1303.

5. Zhang L, Wu Z, Zhou Q. Epithelial-mesenchymal transition and tumor drug resistance. Chin J Lung Canc. 2013;16(1):54-57.

6. Eke I, Cordes N. Focal adhesion signaling and therapy resistance in cancer. Semin Cancer Biol. 2015;31:65-75.

7. Bosquillon C. Drug transporters in the lung-do they play a role in the biopharmaceutics of inhaled drugs? J Pharm Sci. 2010;99(5): 2240-2255.

8. Obexer P, Ausserlechner MJ. X-linked inhibitor of apoptosis protein a critical death resistance regulator and therapeutic target for personalized cancer therapy. Front Oncol. 2014;4:197.

9. Dave B, Mittal V, Tan NM, et al. Epithelial-mesenchymal transition, cancer stem cells and treatment resistance. Breast Cancer Res. 2012; 14(1):202.
10. Espinoza I, Miele L. Deadly crosstalk: Notch signaling at the intersection of EMT and cancer stem cells. Cancer Lett. 2013;341(1):41-45.

11. Assi K, Bergstrom K, Vallance B, et al. Requirement of epithelial integrin-linked kinase for facilitation of Citrobacter rodentium-induced colitis. BMC Gastroenterol. 2013;13:137.

12. Rhee SH, Han I, Lee MR, et al. Role of integrin-linked kinase in osteosarcoma progression. J Orthop Res. 2013;31(10):1668-1675.

13. Chen D, Zhang Y, Zhang X, et al. Overexpression of integrin-linked kinase correlates with malignant phenotype in non-small cell lung cancer and promotes lung cancer cell invasion and migration via regulating epithelial-mesenchymal transition (EMT)-related genes. Acta Histochem. 2013;115(2):128-136.

14. Zhao G, Guo LL, Xu JY, et al. Integrin-linked kinase in gastric cancer cell attachment, invasion and tumor growth. World $J$ Gastroenterol. 2011;17(30):3487-3496.

15. Imanishi $Y, \mathrm{Hu} B$, Jarzynka MJ, et al. Angiopoietin-2 stimulates breast cancer metastasis through the $\alpha 5 \beta 1$ integrin-mediated pathway. Cancer Res. 2007;67(9):4254-4263.

16. Gil D, Ciolczyk-Wierzbicka D, Dulinska-Litewka J, et al. The mechanism of contribution of integrin linked kinase (ILK) to epithelial-mesenchymal transition (EMT). Adv Enzym Regul. 2011;51(1): 195-207.

17. Serrano I, McDonald PC, Lock FE, et al. Role of the integrin-linked kinase (ILK)/Rictor complex in TGFbeta-1-induced epithelialmesenchymal transition (EMT). Oncogene. 2013;32(1):50-60.

18. Metro G, Cappuzzo F, Finocchiaro G, et al. Development of gemcitabine in non-small cell lung cancer: the Italian contribution. Ann Oncol. 2006;17(Suppl 5):v37-v46.

19. Luca T, Federico C. Gemcitabine for the treatment of advanced nonsmall cell lung cancer. Oncol Targets Ther. 2009;2:209-217.

20. Kovalev AA, Tsvetaeva DA, Grudinskaja TV. Role of ABC-cassette transporters (MDR1, MRP1, BCRP) in the development of primary and acquired multiple drug resistance in patients with early and metastatic breast cancer. Exp Oncol. 2013;35(4):287-290.

21. Kohan HG, Boroujerdi M. Time and concentration dependency of P-gp, MRP1 and MRP5 induction in response to gemcitabine uptake in Capan-2 pancreatic cancer cells. Xenobiotica. 2015:1-11.
OncoTargets and Therapy

\section{Publish your work in this journal}

OncoTargets and Therapy is an international, peer-reviewed, open access journal focusing on the pathological basis of all cancers, potential targets for therapy and treatment protocols employed to improve the management of cancer patients. The journal also focuses on the impact of management programs and new therapeutic agents and protocols on

\section{Dovepress}

patient perspectives such as quality of life, adherence and satisfaction. The manuscript management system is completely online and includes a very quick and fair peer-review system, which is all easy to use. Visit http://www.dovepress.com/testimonials.php to read real quotes from published authors. 